\title{
Women's views on overdiagnosis in breast cancer screening: a qualitative study
}

\author{
(c) $(1)(9)$ OPEN ACCESS
}

\author{
Jolyn Hersch PhD candidate ${ }^{12}$, Jesse Jansen research fellow ${ }^{12}$, Alexandra Barratt professor of \\ public health ${ }^{2}$, Les Irwig professor of epidemiology ${ }^{1}$, Nehmat Houssami breast physician and \\ associate professor and principal research fellow ${ }^{1}$, Kirsten Howard professor of health economics ${ }^{1}$, \\ Haryana Dhillon research fellow ${ }^{2}$, Kirsten McCaffery associate professor and principal research \\ fellow $^{12}$
}

${ }^{1}$ Screening and Test Evaluation Program (STEP), School of Public Health, University of Sydney NSW 2006, Australia; ${ }^{2}$ Centre for Medical Psychology \& Evidence-based Decision-making (CeMPED), University of Sydney NSW 2006, Australia

\begin{abstract}
Objective To elicit women's responses to information about the nature and extent of overdiagnosis in mammography screening (detecting disease that would not present clinically during the woman's lifetime) and explore how awareness of overdiagnosis might influence attitudes and intentions about screening.

Design Qualitative study using focus groups that included a presentation explaining overdiagnosis, incorporating different published estimates of its rate $(1-10 \%, 30 \%, 50 \%)$ and information on the mortality benefit of screening, with guided group discussions

Setting Sydney, Australia

Participants Fifty women aged 40-79 years with no personal history of breast cancer and with varying levels of education and participation in screening.

Results Prior awareness of breast cancer overdiagnosis was minimal. Women generally reacted with surprise, but most came to understand the issue. Responses to overdiagnosis and the different estimates of its magnitude were diverse. The highest estimate (50\%) made some women perceive a need for more careful personal decision making about screening. In contrast, the lower and intermediate estimates (1-10\% and $30 \%$ ) had limited impact on attitudes and intentions, with many women remaining committed to screening. For some women, the information raised concerns, not about whether to screen but whether to treat a screen detected cancer or consider alternative approaches (such as watchful waiting). Information preferences varied: many women considered it important to take overdiagnosis into account and make
\end{abstract}

informed choices about whether to have screening, but many wanted to be encouraged to be screened.

Conclusions Women from a range of socioeconomic backgrounds could comprehend the issue of overdiagnosis in mammography screening, and they generally valued information about it. Effects on screening intentions may depend heavily on the rate of overdiagnosis.

Overdiagnosis will be new and counterintuitive for many people and may influence screening and treatment decisions in unintended ways, underscoring the need for careful communication.

\section{Introduction}

Mammography screening is meant to reduce breast cancer mortality through early diagnosis and treatment of disease that would otherwise threaten life. However, in some cases a screening mammogram detects a cancer that would have neither caused death nor presented clinically during the woman's lifetime, ${ }^{1}$ meaning she would never have acquired a cancer diagnosis had she not attended screening. ${ }^{2}$ This is termed overdiagnosis or overdetection. Overdiagnosis and the resulting overtreatment of inconsequential disease are considered the most serious and important harms associated with early detection of cancer through screening, ${ }^{1-3}$ generating morbidity that adds to the societal burden of cancer in medical, psychological, and economic terms. ${ }^{4}$ An overdiagnosed person is unlikely to benefit and may be harmed physically and emotionally by diagnostic procedures and treatments. The implications of the diagnosis 
may extend to female relatives, who are consequently classified as being at higher risk. ${ }^{56}$

The problems of overdiagnosis and overtreatment, both in cancer screening ${ }^{1}$ and in the broader healthcare context, ${ }^{7}$ are receiving increasing attention, and an independent review took place in 2012 in the United Kingdom ${ }^{8}$ to assess whether the national breast screening programme needed to be changed in light of the latest evidence on benefits and harms, including overdiagnosis. Quantifying overdiagnosis in mammography screening remains a challenge-all estimates are subject to bias, and findings vary over a wide range amid continuing methodological debate. ${ }^{9-11}$ Recent studies have suggested that the overdiagnosed proportion of breast cancers (invasive and non-invasive) diagnosed in women invited for screening is likely to be around $19 \%$ to $35 \%,{ }^{8}{ }^{12-15}$ whereas other estimates have been substantially lower ${ }^{16}{ }^{17}$ or higher. ${ }^{18} 19$

Debate persists regarding the appropriateness of informing women about the risk of overdiagnosis before they decide to screen. ${ }^{20}$ Many argue that women considering screening should be aware of benefits and harms, including overdiagnosis, so they can make informed decisions about whether to participate. ${ }^{14{ }^{14-25}}$ However, others are wary of the possibility that explicit information on the downsides of screening might dissuade people from participating. ${ }^{20}{ }^{26}$ Materials distributed by breast screening programmes worldwide generally do not explain overdiagnosis, ${ }^{3527-29}$ and consequently the public is largely unaware of the issue..$^{30-32}$ Two mammography decision aids (evidence based information resources to support decision making) have briefly introduced the concept of overdiagnosis via an optional hyperlink ${ }^{33}$ or appendix..$^{34}$ However, the information was probably not sufficient to convey the full implications of overdiagnosis and overtreatment, including the number of women affected. In a New Zealand study, ${ }^{35}$ a citizens' deliberation method was used to educate a panel of 11 women about mammography benefits and harms, of whom 10 then changed their minds to vote against government provision of screening for women aged 40-49. Although overdiagnosis was referred to repeatedly during group deliberations about screening policy, ${ }^{35}$ it remains unclear how women respond to overdiagnosis on an individual level and how they might integrate this information with their personal attitudes in deciding about their own screening participation. There is also a lack of evidence about how sensitive preferences might be to the varying estimates of overdiagnosis. ${ }^{1}$

Mammography screening involves large numbers of women worldwide, with participants annually numbering over 840000 in Australia ${ }^{36}$ and 2.1 million in the UK. ${ }^{37}$ The Australian mammography programme is similar to those in other developed countries: women are invited to a screening service whose leaflets emphasise the benefit without mentioning overdiagnosis (such as www.bsnsw.org.au/publications/early-detection-isvital). To develop optimal strategies for communicating about overdiagnosis to women considering screening, better understanding of women's perspectives on the issue is needed, ${ }^{38}$ including how views may vary according to the magnitude of overdiagnosis. We conducted a qualitative study to elicit women's responses to information about overdiagnosis in mammography screening. We employed qualitative methods, which have been used successfully in previous research into women's views of screening, their reasoning about participation, and the ideas shaping these decisions. ${ }^{39} 40$ A qualitative approach was well suited to the goals of this study which aimed to generate insights into women's conceptual understanding and interpretation of information on overdiagnosis; to explore women's evaluation of overdiagnosis including whether and how different estimates influenced screening attitudes and intentions; and to investigate views about provision of such information to the community.

\section{Method \\ Design}

We used focus groups to explore how women integrated information about overdiagnosis (a topic likely to be new to them) with their existing understanding of breast screening (or "schema") ${ }^{41}$ and how they might use the information when making their personal screening decisions. Choosing a qualitative design involving face to face contact with participants ensured we could be confident that we communicated the information effectively. Women were able to ask questions to seek clarification, meaning that we could check their understanding and identify sources of confusion as part of the research process. Collecting data through focus groups rather than individual interviews gave more control of conversation topics to the participants because it allowed participants to bring up and discuss among themselves, in their own language, relevant points beyond those anticipated by the researchers. ${ }^{42}$ Another useful feature of focus groups for this research was the opportunity for participants to hear each other's views and exchange ideas, thereby helping to clarify individual understanding and attitudes. ${ }^{43}$ Furthermore, the presence of group members with contrasting opinions often led participants to elaborate their own point of view in greater depth. ${ }^{42}$

All study procedures were approved by the University of Sydney Human Research Ethics Committee. Participants gave written consent prior to participation.

\section{Participant selection and recruitment}

We recruited a community sample of women for whom making decisions about participating in mammography screening was relevant. Eligible women were aged 40-79 years, spanning the broad age range in which women commonly have screening. In Australia, women aged 50-69 are routinely invited for free biennial mammograms through the national screening programme, while women aged $40-49$ or over 70 are eligible for screening on request but not actively invited.

We selected Sydney suburbs that varied in socioeconomic status and approached potential participants by randomly sampling households in these areas using the electronic telephone directory. Recruitment was carried out via telephone by the Hunter Valley Research Foundation (HVRF), an independent non-profit organisation with extensive experience running community surveys and recruiting people into health research. To gain a diverse range of perspectives, we used purposive sampling to ensure inclusion of women with varying levels of education (that is, with and without post-school qualifications) and prior participation in screening (including low and high screening women in all age groups). We excluded women not fluent in English and women who had ever personally been diagnosed with breast cancer. Trained HVRF interviewers used a short series of questions to determine eligibility and compiled a database of women who had consented to contact by the researchers. We mailed study information to these women and then telephoned them to address any questions and invite them to participate.

Of 118 women who were contacted and eligible, 78 (66\%) consented to participate. We scheduled focus group sessions to best accommodate women's availability. Recruitment to new groups continued until the team decided we had achieved 
thematic saturation as indicated by data redundancy — that is, when participants no longer raised themes not already elicited $(\mathrm{n}=50))^{44} 45$

\section{Conducting the focus groups}

We conducted eight age-stratified focus groups, each comprising four to nine women, from July to September 2011. Sessions were convened in neutral focus group facilities distributed across three Sydney locations that were convenient for participants rather than in a clinical or academic setting. Each session comprised several parts: introduction and consent, baseline questionnaire, warm-up discussion, a detailed presentation on breast screening and overdiagnosis which was divided into sections and interspersed with guided discussions about the content, and a final questionnaire. Sessions lasted about two hours and were facilitated by a pair of female moderators $(\mathrm{JH}$ and either JJ or KM, all researchers in public health and psychology). Each participant was paid \$A150 to reimburse her for her time and to cover travel costs. We provided all participants with a written list of organisations from which they could access further information about breast screening if desired.

\section{Presentation and discussion content}

At each session, JH gave an audiovisual presentation that had been developed and reviewed by a multidisciplinary expert team of researchers and clinicians (see appendix 1 of supplementary material on bmj.com for example slides). The presentation explained overdiagnosis using a consumer friendly format (such as combining pictures with text and using plain language as much as possible). We presented a range of published estimates of the rate of overdiagnosis in mammography screening (1-10\%, $30 \%$, and $50 \%$ of breast cancers diagnosed in women participating regularly in screening), based on a thorough literature review (see appendix 2 on bmj.com for further details on these estimates). To put overdiagnosis in context, we also provided age stratified, evidence based information on the benefit in terms of breast cancer mortality associated with mammography screening in Australia. ${ }^{46}$ In line with evidence based recommendations ${ }^{47}$ we illustrated cumulative outcomes in the form of natural frequencies using diagrams to show the absolute number of breast cancer deaths among 1000 screened and 1000 unscreened women, and the number of cases overdiagnosed in the screened cohort. Thorough pilot testing of the study materials and procedure among women in the relevant age range resulted in various refinements (such as replacing the term "overdiagnosis" with "overdetection" to avoid confusion with misdiagnosis).

In the group discussions, women shared their responses to the information presented and how it made them feel about screening, as well as views on different strategies for communicating about screening. Box 1 summarises the presentation content and types of questions we used to guide the discussions. At the beginning of each focus group we made it clear that we would be talking about reasons why women might choose to have screening or not to have screening, and highlighted that we would not try to persuade them one way or another. We emphasised our neutrality throughout by repeating statements of this kind, by not labelling the figures presented (such as number of overdiagnosed cases) as large or small, and by not interpreting as positive or negative any views expressed by participants. Throughout the presentation, we strongly encouraged women to request clarification as often as they needed.

\section{Data capture, coding, and analysis of qualitative data}

Focus group discussions were audio recorded and transcribed verbatim. Data were managed using NVivo 9 software. We conducted a thematic analysis, aiming to identify a set of main themes that captured the diverse views and feelings expressed. After the first few focus groups, the group moderators $(\mathrm{JH}, \mathrm{JJ}$, and $\mathrm{KM}$ ) began the analysis by independently documenting and then discussing together the most salient observations from the group discussions. These impressions, together with the research questions that shaped the group discussion guide, formed the basis of the initial coding framework.

Two researchers ( $\mathrm{JH}$ and $\mathrm{JJ}$ ) independently reviewed all transcripts, developed codes in an iterative process, and applied these to the data to identify patterns of ideas. Using constant comparison, ${ }^{44}{ }^{48}$ we continually looked for similarity and differences in the data and our coding within and across transcripts. By comparing each part of the data with the rest of the data, we established analytical categories and selected key overarching concepts. Throughout this process we met regularly with $\mathrm{KM}$ and the research team to discuss the framework with which to interpret the data.

\section{Quantitative measures}

We administered brief written questionnaires at the start and end of each focus group, including demographics, preferred involvement in decision making about screening (control preferences scale) ${ }^{49}$ and items to assess basic background knowledge and understanding of content presented (see appendix 3 on bmj.com for key items from questionnaires 1 and 2).

\section{Follow-up telephone interviews}

$\mathrm{JH}$ conducted a brief telephone interview with a randomly selected third of the focus group participants in each age category $(n=18)$ at one to four weeks after their session to identify whether views about overdiagnosis had changed and to elicit any responses that women might prefer to express privately. We asked whether the women had any further thoughts or feelings, since their focus group, about the information presented and their screening intentions. As the views expressed during follow-up telephone interviews presented no new data further to the focus group discussions, they were not analysed further.

\section{Results}

\section{Sample characteristics}

Fifty women participated in eight focus groups. Participants had diverse cultural and education backgrounds (table $1 \Downarrow$ ). Each age stratum included women who had participated regularly in screening and others who had not. Three quarters of women in the target 50-69 year old cohort and $60 \%$ of older women knew they were eligible for free screening, whereas two thirds of women in their 40s either believed they were too young to access the programme or were unsure. About $80 \%$ of respondents desired active involvement in decisions about their breast screening participation.

To illuminate the study findings presented below, we have selected representative participant quotations that illustrate both typical responses and the diversity of views expressed. The examples are labelled by participant number, age, and screening history, and are drawn from women with a variety of education levels. 


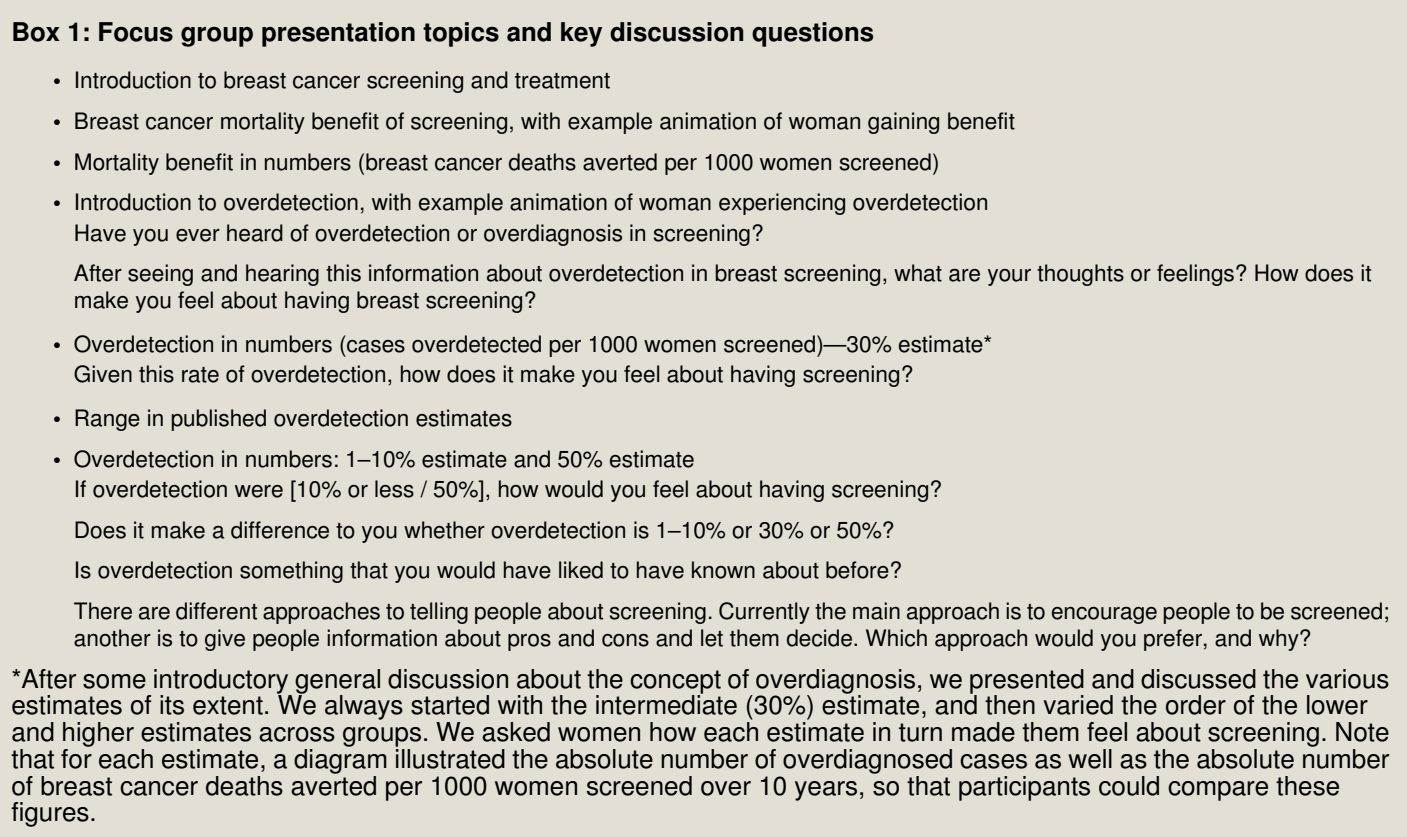

\section{The concept of overdiagnosis: women's understanding and reactions}

\section{Understanding of the concept of overdiagnosis}

Prior awareness of overdiagnosis was limited to only a few women who had heard of it in the context of prostate cancer. The idea of overdiagnosis occurring in breast cancer screening was surprising and challenged women's beliefs about breast cancer generally being a serious and dangerous disease:

'In your brain, breast cancer-if it isn't treated-is fatal. So I've never ever heard of that before, and I think most women wouldn't have." (Participant 26, age 55 years, 3 screens)

"That there are okay cancers, it's something that we haven't heard of before. It's very new to me... here it is... in the statistics, but we don't hear about it, not even from our doctors." (Participant 15, age 47, 1 screen).

By the end of the session, most women gained a reasonable understanding of the concept of overdiagnosis, as shown by these examples of women paraphrasing the concept of indolent or "slow growing" cancers that do not threaten life:

"Sometimes it doesn't matter if you've got [breast cancer], it wouldn't make any difference, you're still going to live as long." (Participant 27, age 57, 0 screens)

"It could be dormant for years and years, and you wouldn't know." (Participant 49, age 78, 4 screens).

We also assessed participants' conceptual knowledge about overdiagnosis via two items in the written questionnaire administered at the end of each focus group. As shown in table $2 \Downarrow$, most of the participants' written responses indicated understanding of the key point that screening increases breast cancer incidence through the detection of non-threatening cancers. We marked women's definitions of overdetection strictly according to the following scoring criteria. To score full marks, the response had to indicate that (a) a person has cancer, and $(b)$ it may not cause harm or treatment may not be necessary. The "partially correct" scoring category covered responses that ranged from those indicating some limited understanding (such as "finding a lump that is benign") to those that were close to achieving full marks (such as "when there is a cancer that is detected, but may have not changed if it was not detected").
The complete set of written definitions given by participants is provided in appendix 4 on bmj.com.

There were many questions around the methods used to identify overdiagnosis. Women often had trouble grasping how it is even possible to know that overdiagnosis is occurring:

"How do we know that things are being overdetected? How do we know that there are some cancers that move more quickly or become more malignant than others? ...

Overdiagnosis assumes that these women, who have been overdiagnosed, have a cancer that is not necessarily aggressive ... Who determines what's an aggressive and a non-aggressive cancer?" (Participant 33, age 62, 5 or more screens).

Many women expressed surprise or disbelief at the current limitations we described in distinguishing between cancers that do require treatment and those that may not. There was some prior awareness that cancers are graded and staged after biopsy, and our assertion that this process still cannot accurately predict the future course of every diagnosed cancer (and the need for treatment) conflicted with women's expectations:

"If you have a lump ... don't they do a biopsy to check whether it is cancerous or not first? Yes and if it is, they would be able to tell you whether they are aggressive or not, right?" (Participant 11, age 46, 2 screens).

Women also struggled with the fact that overdiagnosis cannot be identified at the level of individual patients - so that even if a given case is "slow growing," there is no way to know for sure that it will remain non-threatening throughout the woman's lifetime and thus represents an instance of overdiagnosis:

"How do they find out? ... Have they studied a slow growing breast cancer to see how long it goes for or how do they know that?" (Participant 24, age 52, 1 screen).

Box 2 summarises the main issues around overdiagnosis that caused confusion or required clarification during the focus groups.

\section{Reactions to learning about overdiagnosis}

For some women, learning about overdiagnosis prompted concern about the implications in terms of the undesirable 


\section{Box 2: Summary of main points of confusion about overdiagnosis, based on women's questions}

- How do you know that there are cancers that will not progress fast enough to cause the woman any harm in her lifetime?

- Why is it not possible to distinguish these non-threatening cancers from the potentially dangerous ones that require treatment, even after biopsy or surgical removal of the cancer?

- How can overdiagnosis be quantified if we cannot identify the individual overdiagnosed cases?

- Why does overdiagnosis matter?

psychological and physical consequences of experiencing a cancer diagnosis and enduring treatment unnecessarily:

"It could cause a lot of unnecessary stress and heartache to the person." (Participant 42, age 72, 3 screens)

"So you have to go through all the rigmarole of chemotherapy, radiation, all that sort of thing, and it may not have been necessary, and those in themselves are hard to go through.” (Participant 09, age 45, 0 screens).

In contrast, a few women reacted quite defensively to the concept of overdiagnosis or questioned why it mattered. They disagreed with the notion that it could be considered a bad outcome to find a cancer and have treatment that was ultimately not needed. These women perceived "overdetection" as a value laden term, labelling the phenomenon as negative without real justification. There was also some concern about the potential to upset women who had previously accepted invitations to screening:

'I think the terms 'overtreatment' and 'overdiagnosis' are negative, they're loaded terms, and I don't think it's a necessarily negative occurrence.... Whatever kind of cancer it is-from vanilla nothing through to instant killer-finding that, can that be a negative? I don't think so." (Participant 10 , age 45,1 screen)

“To me it's not 'over' ... I don't believe in 'overservicing' ... I think this is a terrible word to use because I think it's a bullying word that people think they've been pushed into doing something that's not relevant." (Participant 05, age 43, 1 screen).

In several groups, women speculated about ulterior motives behind overdiagnosis research. For example, some wondered whether our presentation might be part of a government plan to reduce spending on breast screening, using overdiagnosis as justification:

"I think what they're trying to do here is cut out breast screening ... or cut out the funding. ... I would hate to see the funding cut for mammograms. I think it's really important that we keep it up, if that's what all this is about." (Participant 34 , age 67,5 or more screens).

In responding to our introduction to the concept of overdiagnosis, only one woman explicitly asked for quantitative information about its frequency of occurrence.

\section{The impact of overdiagnosis information on decision making \\ Implications of overdiagnosis for decisions about screening}

Exploring how awareness of overdiagnosis affected women's feelings about screening was one of our key objectives for the focus group discussions. We found that a few participants, especially younger women with limited screening experience, quickly interpreted the possibility of overdiagnosis and overtreatment as a distinctly negative, off-putting factor in the screening "equation":
"Turns me off further ... I'm just adding the things that we've learnt together ... and all the doctors not knowing if it is malignant or not, you add them all together and you think, 'Why bother?'” (Participant 09, age 45, 0 screens)

"I don't know, makes me a bit scared now to go for a mammogram." (Participant 12, age 46, 0 screens).

After explaining and discussing the concept of overdiagnosis, we moved on to its rate, starting with the $30 \%$ estimate. Although this figure clearly surprised some women and raised a few momentary doubts, most women found this level of overdiagnosis acceptable and considered it to be outweighed by the possible benefits of early detection. Ultimately, the $30 \%$ estimate had limited impact on women's existing views and screening intentions:

"I'm surprised there is overdetection, but I don't think it will really change my view." (Participant 13, age 47, 0 screens)

"It's probably a high number I suppose ... but then, personally, I still think I would prefer to be screened than not." (Participant 38, age 70, 5 or more screens)

"I still think the deaths are high, so I prefer taking the risk of [being] 'overdetected' than ... risk of dying." (Participant 15 , age 47,1 screen).

The $1-10 \%$ overdiagnosis estimate was generally perceived as negligible and nothing to worry about. It did not seem to be sufficient to influence screening decisions but rather tended to confirm women's confidence in screening:

“The 1-10 is like nothing." (Participant 24, age 52, 1 screen)

"If it's in the $10 \%$ range, then I will say, 'Yeah, just go ahead and do it." (Participant 11, age 46, 2 screens).

By contrast, the $50 \%$ overdiagnosis estimate was seen as extremely high. Considering this rate elicited emotional reactions, some bordering on disbelief, and raised concerns about the potential burden of unnecessary treatment affecting so many women:

"Fifty per cent, that's a huge number. ... I do voluntary work at [hospital], and [the patients having cancer treatment] look dreadful and they feel dreadful. ... I hate to think of $50 \%$ of people going through all that awful treatment for no reason." (Participant 42, age 72, 3 screens).

The figure $\Downarrow$ illustrates a range of ways in which women responded to the $50 \%$ overdiagnosis scenario. Some women were prompted to reconsider whether screening was likely to be worthwhile for them personally - that is, taking into account their own perceived risk factors for breast cancer (such as family history, breast feeding, the pill, breast self examination, lifestyle, stress). The discussions suggested that if the rate of overdiagnosis was about $50 \%$, some participants might decline screening altogether. More commonly, women suggested that they might delay screening (particularly younger women) or be less concerned or rigorous in ensuring they attended within the recommended two year interval. Notably, women often presented this as a relatively minor change in their screening intentions. For one woman, the $50 \%$ overdiagnosis estimate had the effect of undermining her confidence in mammography as 
a screening tool and stimulating a search for other screening modalities. However, some women indicated that the information did not at all affect their feelings that mammography screening was worthwhile. A few declared that they would still participate in screening regardless of an even higher overdetection risk, and even while interpreting $50 \%$ as a high rate in itself.

Across the sample, women made a clear distinction between the $30 \%$ and $50 \%$ estimates. Responses suggested that an overdetection rate of $50 \%$ might deter some women from screening at all, and for many others it would at least necessitate more careful thought about whether and when to screen. In other words, the higher rate would affect the decision making process even if it might not change women's ultimate behaviour:

"If there's more chance that you would have all the treatment and it was unnecessary, you'd be less likely to have the screening, I would say." (Participant 30, age 61, 0 screens)

"It does make a difference. ... I'd think about it a lot more with the $50 \%$, whereas $30 \%$ I'd probably do it more easily." (Participant 24, age 52, 1 screen).

We also observed a relationship between previous screening attendance and the extent to which information about overdiagnosis seemed to influence perceptions of screening. Regular screeners tended not to alter their positive screening intentions after learning about overdiagnosis, whereas women with less screening experience seemed more concerned about the issue.

\section{Implications of overdiagnosis beyond screening decisions}

Women valued screening as an opportunity to gain a sense of reassurance that everything was all right. They recognised that, even in light of overdiagnosis, they still had good chances of going through screening over many years without having any cancer diagnosed. Therefore, many women did not seem motivated to make a well informed and carefully considered decision about whether to have screening. Instead they chose to defer engaging in thorough deliberation until they were faced with the relatively unlikely event of being diagnosed with cancer:

"I think screening is ... something you go through to have a peace of mind. But as to ... after that, if you are diagnosed, then you have to weigh and look at the various options that you have." (Participant 11, age 46, 2 screens).

Some women said they would still have screening but felt that, if they were subsequently diagnosed with breast cancer, treatment (or at least adjuvant therapies beyond surgery) may not be worth while if overdiagnosis were $50 \%$. Rather than seeing treatment as the immediate logical consequence of a diagnosis, they would want to consider "watchful waiting" or alternative therapies. At the very least, women anticipated having more thorough discussions and desired greater involvement with their doctors to explore all their management options:

"If you were diagnosed you'd really go into what treatment involves ...you'd want to make a really informed decision ... [but] I probably would still have the breast screens and then make the decision after." (Participant 26, age 55, 3 screens)

"I'll still go [for screening]. ... Surgery's okay too, but ... anything else being needed, any chemicals to be put through my body ... then that's where I stop and think and look at the statistics." (Participant 15, age 47, 1 screen)
"It doesn't turn me off the screening, but I would certainly, probably second-think the treatment. Not that I wouldn't get it done, I probably may take a bit longer to think about it." (Participant 16, age 47, 0 screens).

There were suggestions that awareness of overdiagnosis (that is, knowing that not all cancers progress and need treatment) might help newly diagnosed women to feel less afraid and more hopeful about their prognosis. Some participants felt that this knowledge might also give such women more of a sense of control and reduce the pressure to follow a predetermined disease management pathway.

Importantly though, it was also suggested that awareness of overdiagnosis could worsen the experience of having cancer by making women feel less able to cope with the challenges of unpleasant treatment. As such, it was considered perhaps better for the woman concerned to believe the cancer to be life threatening and treatment crucial:

"That $50 \%$ is not good information ... because if I was told ... I've got cancer, I won't want to have in the back of my mind, 'Oh well, it might not be, and I'm going through all this and my family's all going bananas for nothing."'

(Participant 42, age 72, 3 screens).

\section{How to communicate overdiagnosis information to women}

\section{Personal preferences regarding screening information}

Some respondents stated that the information about overdetection would not make any difference to their own screening views or behaviour and therefore it did not matter whether they had this information or not:

"For me ... if I'm going to do it, I don't care about [the overdetection rate]." (Participant 35, age 69, 5 or more screens).

Others felt that it was good to know about overdiagnosis in order to be able to make informed decisions about screening. This included women who felt that their own screening decision would change and those who thought that it would not:

"I really think it's good to know the downside of overdetection. ... That information [is] not discouraging me to ... screen, but it just made me feel I have knowledge. ... I feel it's really good." (Participant 18, age 49, 0 screens).

Some participants thought that making decisions was more difficult when there was a lot of information to go through, or they lacked confidence in their ability to understand numerical information (such as the chances of experiencing benefit or harm from screening). These women often preferred to trust recommendations from their doctor or the government:

"It's confusing, especially if you're not mathematical, like me." (Participant 34, age 67, 5 or more screens)

"I just want [my doctor] to say, 'You should go, you must go ... this is where you go." (Participant 12, age 46, 0 screens).

Many respondents were in favour of being encouraged to screen, whereas some preferred to be given balanced information and left to make an individual choice. Some women simultaneously endorsed both of these approaches:

"I'd like to be encouraged to have screening. I'd also like to be armed with all the information." (Participant 33, age 62, 5 or more screens). 


\section{Views on providing information to the community}

Many women favoured full, balanced information as the most ethical approach to communicating about screening. Some were surprised that they had not heard about overdetection before and argued that up-to-date information about it ought to be more widely available. Again, this included women who remained positive about undergoing screening:

"I think if you've got the information, it should be provided." (Participant 16, age 47, 0 screens)

"I would actually say that women are entitled to know. ... How you act upon it is probably neither here nor there. It's not a bad thing, perhaps, if that reminder [letter every two years] comes with this information attached to it—or any new information, for that matter, that might arise."

(Participant 17, age 48, 1 screen).

On the other hand, women recognised the challenge posed by trying to communicate the complex information from the focus group presentation in an alternative format that would be accessible and more practical. Furthermore, some women felt that changing the conventional pro screening message to include more balanced information could unleash widespread public confusion:

"They're going to confuse the people. ... All this hard work they've been doing for years, encouraging people to have mammograms ... now you are saying today, 'If you want to you can do it, but see it's your choice, see the percentages and you make your own decision.'... Years and years saying, drumming in, 'You should have this every two years, '... and now you come out and say, 'Look, they are the percentages, think about it if you're going to go and do it.' You are confusing people." (Participant 35, age 69, 5 or more screens).

Some women who said they personally were not put off by the overdiagnosis information nonetheless expressed concern that it would dissuade others from screening. In their view, this could lead to more breast cancer deaths and compromise the gains that screening had achieved for women's health. A couple of participants suggested providing overdiagnosis information to women only if and when they were diagnosed with breast cancer rather than before screening. Some participants took an intermediate position, suggesting that the amount of information women required before screening would vary according to personal preferences and that this variation should be accommodated somehow:

"I don't think a vast wad of information should be pushed on everyone willy-nilly, but people should be made aware that the information is there if they would like it ... this might help you make a decision. But I'd want to be made aware that there was information that I can and should look at, should I choose to." (Participant 29, age 59, 5 or more screens).

\section{Discussion Principal findings}

In this community sample of women eligible for mammography screening, awareness of overdiagnosis of breast cancer was minimal, consistent with earlier quantitative survey research. ${ }^{31} 32$ However, most participants were able to understand the issue after the explanation we presented and the opportunity to have their questions addressed. Although virtually everyone reacted to the information about overdiagnosis with surprise, effects on women's screening attitudes were diverse. Some women expressed a commitment to screening that was unaffected by learning about the possibility of overdiagnosis, while others wanted to take this information into account and give further thought to their screening choices. The screening intentions expressed by these women depended heavily on the magnitude of overdiagnosis, with the highest estimate (50\%) having a distinctly greater impact than both the lower and intermediate estimates (1-10\% and 30\%). Most participants felt that this information was important and should be available to enable women to make informed choices, although many also wanted to be encouraged to have screening.

Importantly, for some participants the information on overdiagnosis caused uncertainty not about whether to have screening but rather whether to have standard treatment for a screen detected cancer or consider alternative approaches (such as watchful waiting or unproven therapies). There was also the suggestion that, for women who were treated for breast cancer, being aware of the possibility of overdiagnosis might make it more difficult to endure unpleasant side effects because of uncertainty about whether treatment was really needed. Learning about overdiagnosis in mammography raised interest in seeking alternative forms of breast screening (such as ultrasound scanning) either instead of or in addition to mammography. Several participants expressed concern about other women inappropriately being deterred from screening altogether because of overdiagnosis. Moreover, some were suspicious about an agenda behind overdiagnosis research-including our own study - fearing a government plan to cut funding for mammography screening.

\section{Strengths and limitations}

The strategy for communicating about breast cancer screening in the UK is currently under review. By examining lay women's perspectives on overdiagnosis, this study makes an important and timely contribution to the debate about the possible consequences and value of providing overdiagnosis information to women invited for screening.

The focus group presentation was carefully developed to be both accurate (that is, evidence based data) and understandable (such as innovative illustrations of the concepts), and was expert reviewed and extensively pilot tested. We presented the cumulative probabilities of different screening outcomes using natural frequencies, in line with evidence based

recommendations. ${ }^{47} \mathrm{We}$ explored responses to a diverse range of overdiagnosis estimates, reflecting the full body of existing literature, because the amount of overdiagnosis is contested and all estimation methods are subject to bias. ${ }^{9}$ The qualitative design is a strength of this exploratory study because it allowed unanticipated findings to emerge (such as the impact on decision making about accepting treatment for detected cancer).

Encouraging women to ask questions also provided new insights into points of confusion.

We tried to minimise volunteer bias by recruiting directly from the general community, and our provision of financial compensation helped us to achieve a broad sample including women from diverse socioeconomic backgrounds who varied in age, education, prior screening, and ethnicity. However, as the sample was not designed to be statistically representative, we cannot conclude that our findings reflect general population views. We stressed our neutrality at the start of each session to mitigate against "socially desirable" comments in favour of screening.

To avoid overloading women with information, we did not talk about the scientific uncertainty surrounding estimates of the mortality benefit, nor did we discuss in detail the chances of 
other relevant screening outcomes (such as false positives and false negatives). Discussing these additional points would have provided a more comprehensive picture of factors relevant to screening and the decision making process, and might have influenced the study results. However, further increasing the complexity of the information provided would have limited the study's capacity to address the key research questions concerning overdiagnosis. It is possible that our choice of presenting the benefit information first may have created a "framing" effect resulting in more positive attitudes, but finding positive attitudes to cancer screening is consistent with previous literature. $^{3132} 34$

In comparison with earlier attempts to introduce the concept of breast cancer overdiagnosis to women, ${ }^{31}{ }^{33} 34$ this study involved a much more thorough explanation of the issue and its magnitude, albeit in a more resource intensive manner. The purpose of the focus groups was not to imitate a "real life" setting but to allow us to explore the process through which women understood and responded to information about overdiagnosis, thereby providing valuable insight into women's attitudes. In our diverse sample (including women with low education and non-English speaking backgrounds) most participants' written definitions of overdetection showed full $(60 \%)$ or partial $(24 \%)$ understanding of the concept. However, some participants (several of whom, though reasonably fluent in English, were from other language backgrounds) did not demonstrate a clear understanding of overdiagnosis, which limited our ability to accurately gather their views about it. For such women, an individual counselling and interview session may be a more appropriate way to explain the issues and examine their responses. Importantly, in this study we asked women about their own screening intentions as opposed to their views on national screening policy. ${ }^{35}$ Earlier qualitative research with women diagnosed through screening found that they considered it important to take into account, when making screening decisions, the possibility of being diagnosed with an ultimately harmless condition..$^{25}$ Our study showed that this view, expressed by breast cancer patients whose perspective on screening would have been coloured by their personal experience of cancer, was shared by many women in the general invited population.

\section{Implications and future research}

This study provides important insights into lay interpretations of information about overdiagnosis, with implications regarding how best to inform people about the issue. Firstly, the magnitude of overdiagnosis matters, with many women indicating that their screening behaviour may change depending on the trade-off between the chances of benefit and harm. ${ }^{50}$ The wide variation in published estimates (1\% to $50 \%$ ) is therefore problematic. Additionally, the suggestions from some women that overdiagnosis would be relevant to their decisions only if they were actually diagnosed with a screen detected cancer reveal a concerning misconception that a screening mammogram is a separable event from the cascade of investigation and intervention that may be triggered by an abnormal result. This highlights the need to explain clearly to women that once cancer is detected, evidence based treatment is virtually always indicated because potentially threatening cases cannot be differentiated from those representing overdiagnosis.

Communicators must also take care not to create a situation in which women think they can avoid overdiagnosis by choosing a different screening method (for which evidence is limited) or by supplementing screening mammography with additional testing, which may increase the potential for overdiagnosis.
Thus the ethical imperative to provide information about overdiagnosis must be balanced with the responsibility to address misconceptions that may lead to problems in clinical practice.

Another important point to consider in developing strategies for communication about overdiagnosis relates to understanding how people interact with new information. A theoretical framework used in the field of education known as schema theory ${ }^{41}$ highlights that the way people engage with new material is influenced by their "schema" (existing topic knowledge and expectations). In the case of mammography screening, attitudes tend to be strongly favourable, which is partly a function of the historical and cultural context. Women's attitudes will have been shaped through the information presented to them directly by screening services as well as their broader experience of many years of widespread public health campaigns focused on promoting the benefits of screening without explaining the harm of overdiagnosis. ${ }^{27} 2932$ The very existence of overdiagnosis runs contrary to prevailing notions of cancer and screening. Therefore it is reasonable to expect that new messages about overdiagnosis will not necessarily be immediately understood by everyone and may provoke confusion or scepticism, as we found to some extent in our study. Rather than wishing to be informed in detail about the range of possible outcomes of screening, some participants expressed a preference to receive more basic information together with encouragement to have screening. Such preferences are consistent with the "consider an offer" approach, ${ }^{51}$ in which screening is offered with summarised key information and people are assisted to evaluate whether taking up the offer is right for them. At the same time, people can be encouraged to consider whether they want to access more detailed information that may help them make a choice. ${ }^{51}$ Public views and information needs may change over time as more balanced screening information and increased awareness of harms contribute to changing the schema of expectations people have about screening. Moreover, the diversity we found in women's responses to overdiagnosis supports the need for information providers to tackle the challenge of developing ways to deliver screening information flexibly rather than assuming that women's needs are homogenous.

Given that doctors' recommendations are likely to influence screening behaviour, ${ }^{52}$ one avenue for future research may be to examine the perspectives of relevant clinicians, both in terms of their views on overdiagnosis and their responses to women's attitudes as reported here. However, in countries such as Australia and the UK women are invited directly by the screening service and attend without necessarily having any discussion with a healthcare provider. Therefore, priorities for future research are to investigate whether a meaningful explanation of overdiagnosis can be condensed into a format that could be distributed with screening invitations, and to assess the impact of such information on screening intentions and participation. Also critical is clarification of the magnitude of overdiagnosis, though it is evident that such data cannot necessarily resolve whether screening benefits outweigh harms for any individual as this ultimately requires a value judgement. ${ }^{546}{ }^{50}$ The question remains, how do we best inform women and support them to make informed decisions about mammography screening using the available evidence. ${ }^{23}$

\section{Conclusions}

Overdiagnosis was unfamiliar to a diverse sample of women eligible for mammography screening in Australia. None the less, we found it was possible to explain the issue in such a way that most participants understood the concept and its 
implications. Women's screening preferences were sensitive to the estimated level of overdiagnosis, underscoring the need for urgent efforts to clarify this issue. Critically, women differed in the way they evaluated the relevance of overdiagnosis in making their personal decisions about screening. Some felt that the new information was unlikely to change their existing attitudes and intentions; others considered it important to take the risk of overdiagnosis into account, particularly at higher estimates. Many women expressed a preference for this information to be more widely available. However, their responses to being informed about overdiagnosis also revealed a range of potentially unwanted consequences that communicators must address. Finally, the diversity of views elicited in this study highlights the inappropriateness of making screening policy and communication decisions based on speculative assumptions about what women want and think regarding overdiagnosis. Instead, it is vital that research efforts continue to better understand women's values about overdiagnosis, to clarify its influence on their behaviour, and to determine the optimal methods to support informed choice in mammography screening.

We thank Bruce Armstrong for helpful comments on the draft presentation, the Hunter Valley Research Foundation for recruitment services, Sara Young for transcription services, Paul Glasziou and Jenny Doust for helpful comments on the presentation slides, Lucie Rychetnik and Stacy Carter for helpful discussion regarding the analysis, Ray Moynihan for helpful comments on the draft manuscript, and all study participants.

Contributors: $\mathrm{KM}$ conceived the study. $\mathrm{KM}, \mathrm{AB}, \mathrm{JH}, \mathrm{JJ}, \mathrm{LI}, \mathrm{KH}$, and $\mathrm{NH}$ were involved in designing the study and developing the methods. $\mathrm{JH}$, $\mathrm{KM}, \mathrm{AB}, \mathrm{JJ}$, and $\mathrm{LI}$ obtained funding. $\mathrm{JH}$ coordinated the running of the study and conducted the focus groups, together with $\mathrm{JJ}$ and $\mathrm{KM}$. JH, $\mathrm{JJ}$, and $\mathrm{KM}$ read transcripts, developed the analytical framework, and contributed to the analysis. JH drafted the manuscript. All authors contributed to the interpretation of the analysis and critically revised the manuscript. All authors are guarantors.

Funding: This work was supported by a fellowship grant (No 0189-1) awarded to $\mathrm{JH}$ from the Informed Medical Decisions Foundation and a grant (No 633003) awarded to the Screening and Test Evaluation Program from the National Health and Medical Research Council of Australia. The funders had no role in the design or conduct of the study; in the collection, analysis, and interpretation of the data; or in the preparation or approval of the manuscript.

Competing interests: All authors have completed the ICMJE uniform disclosure form at www.icmje.org/coi_disclosure.pdf (available on request from the corresponding author) and declare: no support from any organisation for the submitted work; no financial relationships with any organisations that might have an interest in the submitted work in the previous three years; no other relationships or activities that could appear to have influenced the submitted work.

Ethical approval: The University of Sydney human research ethics committee approved this study (No 13085). Each participant gave signed consent immediately before the focus group.

Data sharing: No additional data available.

Welch HG, Black WC. Overdiagnosis in cancer. J Natl Cancer Inst 2010;102:605-13. Jorgensen KJ, Gotzsche PC. Who evaluates public health programmes? A review of the NHS Breast Screening Programme. J R Soc Med 2010;103:14-20.

3 Gummersbach E, Piccoliori G, Zerbe CO, Altiner A, Othman C, Rose C, et al. Are women getting relevant information about mammography screening for an informed consent: a critical appraisal of information brochures used for screening invitation in Germany, Italy, Spain and France. Eur J Public Health 2010;20:409-14.

4 McPherson K. Screening for breast cancer: balancing the debate. BMJ 2010;340:c3106.

5 Heath I. It is not wrong to say no. BMJ 2009;338:b2529.

6 Thornton H. Pairing accountability with responsibility: the consequences of screening 'promotion'. Med Sci Monit 2001;7:531-3.

7 Moynihan R, Doust J, Henry D. Preventing overdiagnosis: how to stop harming the healthy BMJ 2012;344:e3502.
8 Independent UK Panel on Breast Cancer Screening. The benefits and harms of breast cancer screening: an independent review. Lancet 2012;380:1778-86.

9 Biesheuvel C, Barratt A, Howard K, Houssami N, Irwig L. Effects of study methods and biases on estimates of invasive breast cancer overdetection with mammography screening: a systematic review. Lancet Oncol 2007;8:1129-38.

10 Wu D, Perez A. A limited review of over diagnosis methods and long-term effects in breast cancer screening. Oncol Rev 2011:5:143-7.

11 Puliti D, Duffy SW, Miccinesi G, de Koning H, Lynge E, Zappa M, et al. Overdiagnosis in mammographic screening for breast cancer in Europe: a literature review. J Med Screen 2012;19(suppl 1):42-56.

12 Kalager M, Adami H-O, Bretthauer M, Tamimi RM. Overdiagnosis of invasive breast cancer due to mammography screening: results from the Norwegian screening program. Ann Intern Med 2012;156:491-9.

13 Bleyer A, Welch $\mathrm{HG}$. Effect of three decades of screening mammography on breast-cancer incidence. N Engl J Med 2012;367:1998-2005.

14 Morrell S, Barratt A, Irwig L, Howard K, Biesheuvel C, Armstrong B. Estimates of overdiagnosis of invasive breast cancer associated with screening mammography. Cancer Causes Control 2010;21:275-82.

15 Jorgensen KJ, Gotzsche PC. Overdiagnosis in publicly organised mammography screening programmes: systematic review of incidence trends. BMJ 2009:339:b2587.

16 De Gelder R, Heijnsdijk EAM, van Ravesteyn NT, Fracheboud J, Draisma G, de Koning HJ. Interpreting overdiagnosis estimates in population-based mammography screening. Epidemiol Rev 2011;33:111-21.

17 Puliti D, Zappa M, Miccinesi G, Falini P, Crocetti E, Paci E. An estimate of overdiagnosis 15 years after the start of mammographic screening in Florence. Eur $\mathrm{J}$ Cancer 2009;45:3166-71.

18 Junod B, Zahl P-H, Kaplan RM, Olsen J, Greenland S. An investigation of the apparent breast cancer epidemic in France: screening and incidence trends in birth cohorts. BMC Cancer 2011;11:401.

19 Zahl P-H, Strand BH, Maehlen J. Incidence of breast cancer in Norway and Sweden during introduction of nationwide screening: prospective cohort study. BMJ2004:328:921-4.

20 Duffy SW, Tabar L, Chen TH, Yen AM, Dean PB, Smith RA. What information should be given to women invited for mammographic screening for breast cancer? Women's Health 2006;2:829-33.

21 Barratt A, Trevena L, Davey HM, McCaffery K. Use of decision aids to support informed choices about screening. BMJ 2004;329:507-10.

22 Bell RJ, Burton RC. Do the benefits of screening mammography outweigh the harms of overdiagnosis and unnecessary treatment? - No. Med J Aust 2012;196:17.

23 Elmore JG. Fletcher SW. Overdiagnosis in breast cancer screening: time to tackle an underappreciated harm. Ann Intern Med 2012;156:536-37.

$24 \mathrm{McPherson} \mathrm{K}$. Publicity of NHS breast cancer screening programme is unfair. $B M J$ 2011;342:d791.

25 Prinjha S, Evans J, McPherson A. Women's information needs about ductal carcinoma in situ before mammographic screening and after diagnosis: a qualitative study. $J$ Med Screen 2006;13:110-4.

26 Bekker HL. Decision aids and uptake of screening. BMJ 2010;341:c5407.

27 Jorgensen KJ, Gotzsche PC. Content of invitations for publicly funded screening mammography. BMJ 2006;332:538-41.

28 Saalasti-Koskinen U, Makela M, Saarenmaa I, Autti-Ramo I. Personal invitations for population-based breast cancer screening. Acad Radiol 2009;16:546-50.

29 Zapka JG, Geller BM, Bulliard J-L, Fracheboud J, Sancho-Garnier H, Ballard-Barbash R, et al. Print information to inform decisions about mammography screening participation in 16 countries with population-based programs. Patient Educ Couns 2006;63:126-37.

30 Collins K, Winslow M, Reed MW, Walters SJ, Robinson T, Madan J, et al. The views of older women towards mammographic screening: a qualitative and quantitative study. $\mathrm{Br}$ $J$ Cancer 2010;102:1461-7.

31 Schwartz LM, Woloshin S, Sox HC, Fischhoff B, Welch HG. US women's attitudes to false positive mammography results and detection of ductal carcinoma in situ: cross sectional survey. BMJ 2000;320:1635-40.

32 Schwartz LM, Woloshin S, Fowler FJ Jr, Welch HG. Enthusiasm for cancer screening in the United States. JAMA 2004;291:71-8.

33 Mathieu E, Barratt AL, McGeechan K, Davey HM, Howard K, Houssami N. Helping women make choices about mammography screening: an online randomized trial of a decision aid for 40-year-old women. Patient Educ Couns 2010;81:63-72.

34 Mathieu E, Barratt A, Davey HM, McGeechan K, Howard K, Houssami N. Informed choice in mammography screening: a randomized trial of a decision aid for 70 -year-old women. Arch Intern Med 2007;167:2039-46.

35 Paul C, Nicholls R, Priest P, McGee R. Making policy decisions about population screening for breast cancer: the role of citizens' deliberation. Health Policy 2008:85:314-20.

36 Australian Institute of Health and Welfare. BreastScreen Australia monitoring repor 2008-2009. Cancer Series No 63. Cat No CAN 60. AlHW, 2011.

37 NHS Breast Screening Programme. Annual review 2011. NHSBSP, 2011.

38 Hersch J, Jansen J, Irwig L, Barratt A, Thornton H, Howard K, et al. How do we achieve informed choice for women considering breast screening? Prev Med 2011;53:144-6.

39 Griffiths F, Bendelow G, Green E, Palmer J. Screening for breast cancer: medicalization, visualization and the embodied experience. Health 2010;14:653-68.

40 Willis K, Baxter J. Trusting technology: women aged $40-49$ years participating in screening for breast cancer-an exploratory study. Aust NZ J Public Health 2003;27:282-6.

41 Anderson RC, Pearson PD. A schema-theoretic view of basic processes in reading comprehension. In: Pearson PD, ed. Handbook of reading research . Longman, 1984:255-91.

42 Wilkinson S. Focus groups in feminist research: power, interaction, and the co-construction of meaning. Women Stud Int Forum 1998;21:111-25.

43 Ritchie J, Lewis J, eds. Qualitative research practice: a guide for social science students and researchers. Sage, 2003.

44 Bowen GA. Naturalistic inquiry and the saturation concept: a research note. Qualitat Res 2008;8:137-52.

45 Kuper A, Lingard L, Levinson W. Critically appraising qualitative research. $B M J$ 2008;337:a1035

46 Barratt A, Howard K, Irwig L, Salkeld G, Houssami N. Model of outcomes of screening mammography: information to support informed choices. BMJ 2005;330:936.

47 Akl EA, Oxman AD, Herrin J, Vist GE, Terrenato I, Sperati F, et al. Using alternative statistical formats for presenting risks and risk reductions. Cochrane Database Syst Rev 2011:CD006776. 


\section{What is already known on this topic}

Mammography screening for breast cancer carries a risk of overdiagnosis (detection of disease that would not present clinically within the woman's lifetime), but estimates of the magnitude of this risk vary widely

Public awareness about breast cancer overdiagnosis is low, and there is little evidence about how women respond to the concept and extent of overdiagnosis and how they might use this information in making personal choices about screening

\section{What this study adds}

Evaluations of the relevance of overdiagnosis depended heavily on the different estimates of magnitude: the $50 \%$ estimate generated substantial concern, whereas the $1-10 \%$ and $30 \%$ estimates were considered more acceptable levels of risk

Women's responses were diverse: some indicated that they would take the risk of overdiagnosis into account when making screening decisions, whereas others felt that their attitudes and intentions to screen were unaffected by the new information

Learning about overdiagnosis raised interest in alternative screening methods as well as concern about whether and how to treat screen detected cancers; hence great care is needed in communicating this information as it may influence screening and treatment decisions in unintended ways

48 Pope C, Ziebland S, Mays N. Qualitative research in health care. Analysing qualitative data. BMJ 2000;320:114-6

49 Degner LF, Sloan JA, Venkatesh P. The control preferences scale. Can J Nurs Res 1997:29:21-43.

50 Welch HG. Overdiagnosis and mammography screening. BMJ 2009;339:b1425.

51 Entwistle VA, Carter SM, Trevena L, Flitcroft K, Irwig L, McCaffery K, et al. Communicating about screening. BMJ 2008;337:a1591.

52 Schonberg MA, McCarthy EP, York M, Davis RB, Marcantonio ER. Factors influencing elderly women's mammography screening decisions: implications for counseling. $B M C$ Geriatrics 2007;7:26.

\section{Accepted: 18 December 2012}

\section{Cite this as: BMJ 2013;346:f158}

This is an open-access article distributed under the terms of the Creative Commons Attribution Non-commercial License, which permits use, distribution, and reproduction in any medium, provided the original work is properly cited, the use is non commercial and is otherwise in compliance with the license. See: http://creativecommons.org/licenses/bync/2.0/ and http://creativecommons.org/licenses/by-nc/2.0/legalcode. 


\section{Tables}

\section{Table 1 | Characteristics of women in focus groups about mammography screening and overdiagnosis $(\mathbf{n}=50)$}

\section{Characteristics}

Age (years)

\begin{tabular}{|c|c|}
\hline $40-49$ & $19(38)$ \\
\hline $50-69$ & $16(32)$ \\
\hline $70-79$ & $15(30)$ \\
\hline \multicolumn{2}{|l|}{ Mammography screening history*: } \\
\hline Screened at least once & $31(62)$ \\
\hline Never screened (age <50 years) & $12(24)$ \\
\hline Never screened (age >50 years) & $7(14)$ \\
\hline \multicolumn{2}{|l|}{ Educationt: } \\
\hline No formal qualifications & $4(8)$ \\
\hline Intermediate school certificate & $11(22)$ \\
\hline Higher school certificate & $9(18)$ \\
\hline Diploma or trade certificate & $12(24)$ \\
\hline University degree & $13(26)$ \\
\hline \multicolumn{2}{|l|}{ Country of birth: } \\
\hline Australia & $27(54)$ \\
\hline England or Scotland & $4(8)$ \\
\hline China & $4(8)$ \\
\hline Other & $15(30)$ \\
\hline \multicolumn{2}{|l|}{ Marital statust: } \\
\hline Married or living with partner & $36(72)$ \\
\hline Single, separated, or widowed & $13(26)$ \\
\hline \multicolumn{2}{|l|}{ No of childrent: } \\
\hline 0 & $11(22)$ \\
\hline 1 & $7(14)$ \\
\hline $2-6$ & $31(62)$ \\
\hline \multicolumn{2}{|l|}{ Employment†: } \\
\hline Retired & $15(30)$ \\
\hline Working full time & $14(28)$ \\
\hline Working part time & $13(26)$ \\
\hline Not in paid work & $7(14)$ \\
\hline \multicolumn{2}{|l|}{ Preferred role in screening decision†: } \\
\hline I decide alone & $13(26)$ \\
\hline I decide after consulting doctor & $12(24)$ \\
\hline Doctor and I share equally in deciding & $14(28)$ \\
\hline Doctor decides after consulting or alone & $8(16)$ \\
\hline
\end{tabular}

*Although the Australian national mammography screening programme is available to women over 40 years old with no upper age limit (so all study participants were eligible), the programme actively recruits women for biennial screening between the ages of 50 and 69 in accordance with current recommendations.

†Missing data for between one and three participants. 
Table 2| Participants' responses to written knowledge items completed at the end of focus group session about mammography screening and overdiagnosis $(n=50)$

Item

No (\%) of women

Who is more likely to be diagnosed with breast cancer? (multiple choice):

\begin{tabular}{lc}
\hline Women who have screening mammograms (correct answer) & $35(70)$ \\
\hline Women who do not have screening mammograms & $10(20)$ \\
\hline Unsure & $5(10)$ \\
\hline What is meant by the term "overdetection"? (free response question): \\
\hline Full marks & $30(60)$ \\
\hline Partially correct & $12(24)$ \\
\hline Incorrect & $8(16)$ \\
\hline
\end{tabular}




\section{Figure}

\begin{tabular}{|c|c|}
\hline $\begin{array}{l}\text { May decline breast } \\
\text { screening entirely }\end{array}$ & $\begin{array}{l}\text { Example: "I probably think, 'Oh yeah, } 50 \% \text { is a high number, and I } \\
\text { won't do it"” (Participant 01, age } 40,1 \text { screen) }\end{array}$ \\
\hline $\begin{array}{l}\text { Seek other forms } \\
\text { of breast cancer } \\
\text { screening }\end{array}$ & $\begin{array}{l}\text { Example: “I may think about trying another sort of way of testing or } \\
\text { checking, because I think, } 50 \% \text {, it’s really unreliable" } \\
\text { (Participant } 18 \text {, age } 49,0 \text { screens) }\end{array}$ \\
\hline $\begin{array}{l}\text { Reconsider in } \\
\text { light of personal } \\
\text { risk factors }\end{array}$ & $\begin{array}{l}\text { Example: "You'd have to look at your general health, your history. ... } \\
\text { If I knew I had a good healthy lifestyle all along, I'd be inclined to say, } \\
\text { 'No, I wouldn't do it'" (Participant } 22 \text {, age } 50,0 \text { screens) }\end{array}$ \\
\hline $\begin{array}{l}\text { Delay screening } \\
\text { or rescreening }\end{array}$ & $\begin{array}{l}\text { Example: "I may delay it a little bit more but probably not too much" } \\
\text { (Participant } 13 \text {, age } 47,0 \text { screens) }\end{array}$ \\
\hline $\begin{array}{l}\text { Views unchanged } \\
\text { - would } \\
\text { still screen }\end{array}$ & $\begin{array}{l}\text { Example: "Doesn't change anything for me. It wouldn't matter how } \\
\text { high it was ... I'd rather be safe than sorry. I think any death is one } \\
\text { death too many" (Participant } 34 \text {, age } 67,5 \text { or more screens) }\end{array}$ \\
\hline
\end{tabular}

Women's responses to a rate of $50 \%$ overdiagnosis for mammography screening 